\title{
HYPERSURFACES OF NONNEGATIVE CURVATURE IN A HILBERT SPACE
}

\author{
BY \\ LEO JONKER
}

\begin{abstract}
In this paper we prove the following generalizations of known theorems about hypersurfaces in $\mathrm{R}^{n}$ : Let $M$ be a hypersurface in a Hilbert space. (1) If on $M$ the sectional curvature $K(\sigma)$ is nonnegative for every 2-plane section $\sigma$ and if $K(\sigma)>0$ for at least one $\sigma$, then $M$ is the boundary of a convex body. (2) If $K(\sigma)=0$ for all $\sigma$, then $M$ is a hypercylinder. The main tool in these theorems is Smale's infinite dimensional Sard's theorem.
\end{abstract}

1. Introduction. Throughout this paper $M$ denotes a Riemannian Hilbert manifold; that is, $M$ is a $C^{\infty}$ connected manifold, modelled on a separable Hilbert space, such that for each $x \in M$ there exists an inner product $\langle\cdot, \cdot\rangle_{x}$ in $M_{x}$, the tangent space of $M$ at $x$, which varies differentiably with $x$. A precise definition may be found in [4]. $M$ can be made into a metric space by letting the distance $d(x, y)$ between two points $x$ and $y$ be the infimum of the lengths of differentiable curves joining them. $M$ is said to be complete if it is complete in this metric.

$A C^{\infty}$ map $\xi: M \rightarrow H$ of a Riemannian Hilbert manifold $M$ into a Hilbert space $H$ is an immersion if the differential $d \xi_{x}: M_{x} \rightarrow H$ is one-to-one and $d \xi_{x}\left(M_{x}\right) \subset H$ is closed in $H$. If $\xi$ is a homeomorphism onto its image, $\xi$ is called an embedding.

An isometric immersion is an immersion $\xi: M \rightarrow H$ such that $d \xi_{x}: M_{x} \rightarrow H$ is an isometry at each point $x \in M$. If in addition $d \xi_{x}\left(M_{x}\right) \subset H$ has codimension one, we call $M$ a bypersurface in $H$. In this case we do not assume that $\xi$ is oneto-one.

Just as in the case of finite dimensional manifolds, the metric on $M$ induces a unique covariant derivative, and we may define the curvature tensor, geodesics, and sectional curvatures. This has been done in [5] where it is also shown that every point has a convex neighbourhood and that completeness implies geodesic completeness.

So far, relatively little is known about the global differential geometry of Riemannian Hilbert manifolds, the most interesting exception being perhaps M. P. doCarmo's partial generalization of a theorem of Sacksteder ([1], [7]). In his paper, doCarmo shows that, if $\xi$ immerses $M$ isometrically in $H$ as a hypersurface, and if at each $x \in M$ all sectional curvatures are positive and bounded away

Received by the editors November 8, 1970.

AMS 1970 subject classifications. Primary 58B20; Secondary 53C40.

Key words and phrases. Hilbert manifold, hypersurface, positive curvature, convex, Sard's theorem, transverse, cylinder, Fredholm map. 
from zero by a positive constant $\delta_{x}$ depending only on $x$, then $\xi$ is an embedding and $\xi(M)$ is the boundary of a convex body in $H$. The hypothesis of doCarmo's theorem is a good deal stronger than that used by Sacksteder, who for a hypersurface in $\mathbf{R}^{n}$ gets the same conclusion by assuming only that all sectional curvatures are nonnegative, and that there is at least one section at one point at which the sectional curvature is strictly positive.

In this paper we will present a method for reducing this kind of theorem about hypersurfaces in Hilbert space to theorems about hypersurfaces in $\mathbf{R}^{n}$. The idea is very simply to intersect the given hypersurface $\xi: M \rightarrow H$ with finite dimensional linear submanifolds $L$ of $H$. Using Smale's infinite dimensional version of Sard's theorem [8], we show in $\$ 2$ that for almost all choices of $L, M$ and $L$ are transverse (Theorems $2.6,2.7$ ). After proving some technical results in $\S 3$, we show in $\S 4$ that, if $L$ and $M$ are transverse, $\xi^{-1}(L)$ is a submanifold of $M$ whose sectional curvatures have the same sign as the sectional curvatures of the corresponding sections of $M$.

In $\$ 5$ we apply our method to obtain the infinite dimensional version of Sacksteder's theorem with the weakest possible hypothesis (Theorem 5.1).

In $\$ 6$ we apply our method to obtain the infinite dimensional counterpart of a theorem of Pogorelov [6] and Hartman and Nirenberg [2] which states that, if the hypersurface $M$ has zero sectional curvatures, $\xi(M)$ is a hypercylinder (Theorem 6.1).

2. Transverse linear sections. Throughout this paper $H$ will denote a fixed separable Hilbert space, and $M$ will denote a Riemannian Hilbert manifold immersed in $H$ as a hypersurface by an isometric immersion $\xi$. We will use $M_{x}$ to denote the tangent space to $M$ at $x$ and $T_{x}$ to denote the linear subspace $d \xi\left(M_{x}\right)$ $C H$.

By a linear submanifold of $H$ we mean a set of points of the form

$$
L+p=\{q+p \mid q \in L\}
$$

where $L$ is any linear subspace of $H$ and $p$ is a point in $H$. If $\left\{L_{1}, \cdots, L_{k}\right\}$ is a set of linear submanifolds of $H$ we shall use $\left[L_{1}, \ldots, L_{k}\right]$ to denote its linear bull; that is,

$$
\left[L_{1}, \ldots, L_{k}\right]=\left\{p \in H \mid p=\sum_{i=1}^{k} \lambda^{i} p_{i}, p_{i} \in L_{i}, \sum_{i=1}^{k} \lambda^{i}=1\right\} .
$$

Occasionally we will also use $\left|\left[L_{1}, \ldots, L_{k}\right]\right|$ to denote its convex bull; that is,

$$
\left|\left[L_{1}, \ldots, L_{k}\right]\right|=\left\{p \in H \mid p=\sum_{i=1}^{k} \lambda^{i} p_{i}, p_{i} \in L_{i}, \sum_{i=1}^{k} \lambda^{i}=1, \lambda^{i} \geq 0\right\} .
$$


If $L_{1}, \cdots, L_{k}$ are all finite dimensional, the set $\left\{L_{1}, \cdots, L_{k}\right\}$ is said to be in general position whenever

$$
\operatorname{dim}\left[L_{1}, \ldots, L_{k}\right]=\sum_{i=1}^{k} \operatorname{dim} L_{i}+(k-1) .
$$

Throughout this paper we will use $G(H, k)$ to denote the space of all linear $k$ manifolds in $H(k<\infty)$, and if $N \in G(H, n), n<k$, we will let $G(H, k, N)$ denote the space of all linear $k$-submanifolds of $H$ that contain $N$. We regard $\emptyset$ as a linear ( - 1)-manifold in $H$; so we identify $G(H, k, \emptyset)=G(H, k)$.

Now let $L_{0} \in G(H, k)$ pass through the origin of $H$, let $K$ be the orthogonal complement of $L_{0}$ and let $\Pi: H \rightarrow L_{0}$ and $\bar{\Pi}: H \rightarrow K$ be the orthogonal projections. Let

$$
V\left(L_{0}\right)=\{L \in G(H, k)|\mathbf{I}| L \text { is one-to-one }\} .
$$

It is clear that these sets cover $G(H, k)$. We may then define a coordinate map on $V\left(L_{0}\right)$ depending on a fixed basis $v_{1}, \cdots, v_{k}$ for $L_{0}$ :

$$
\phi: V\left(L_{0}\right) \rightarrow K^{k+1}
$$

is defined by

$$
\phi(L)=\left(\bar{\Pi}(\Pi \mid L)^{-1}(0), \bar{\Pi}(\Pi \mid L)^{-1}\left(v_{1}\right), \ldots, \bar{\Pi}(\Pi \mid L)^{-1}\left(v_{k}\right)\right) .
$$

The following lemma may be proved by a straightforward computation:

Lemma 2.1. $G(H, k)$ is a $C^{\infty}$ manifold with the structure given by (1). Moreover, if $\emptyset \neq N_{1} \subset N_{2}, G\left(H, k, N_{2}\right)$ is an embedded $C^{\infty}$ submanifold of $G\left(H, k, N_{1}\right)$; and $G\left(H, k, N_{1}\right)$, of $G(H, k)$.

Whenever for $L \in G(H, k)$ and $\xi(x) \in L$ we have $(L-\xi(x))+T_{x}=H$ we will say that $L$ and $M$ are transverse at $x$ (or at $\xi(x)$ ).

For any $N \in G(H, n), n<k$, let $S(H, k, N) \subset H^{k-n}$ be the set of $(k-n)$-tuples $\left(p_{n+1}, \cdots, p_{k}\right) \in H^{k-n}$ such that $\left\{N, p_{n+1}, \cdots, p_{k}\right\}$ is in general position. We will also write $S(H, k)$ for $S(H, k, \emptyset)$. This will be standard notation throughout this paper. We also introduce $\Lambda: S(H, k, N) \rightarrow G(H, k, N)$ defined by

$$
\Lambda\left(p_{n+1}, \cdots, p_{k}\right)=\left[N, p_{n+1}, \cdots, p_{k}\right] \text {. }
$$

The following lemma is easy to prove:

Lemma 2.2. If $N \in G(H, n), k<n$, then $S(H, k, N)$ is a fibre bundle over $G(H, k, N)$ with projection $\Lambda$ and a fibre of dimension $k(k-n)$. Thus $\Lambda: S(H, k, N)$ $\rightarrow G(H, k, N)$ is a Fredbolm map of index $k(k-n)$.

A $C^{\infty}$ map $\Lambda$ between $C^{\infty}$ manifolds is called a Fredholm map if at each 
point the image of $d \Lambda$ is closed and if $\operatorname{dim} \operatorname{ker} d \Lambda$ and codimim $d \Lambda$ are finite. The index at a point is then given by

$$
\text { index } \Lambda=\operatorname{dim} \operatorname{ker} d \Lambda-\operatorname{codim} \operatorname{im} d \Lambda \text {. }
$$

Corollary 2.3. Let $L \in G(H, k, N)$ contain points $p_{1}, \cdots, p_{r}$. Then for a given $\delta>0$ there exists a neighbourbood $V$ of $L$ in $G(H, k, N)$ so that, if $L^{\prime} \epsilon$ $V, L^{\prime}$ contains points $p_{1}^{\prime}, \cdots, p_{r}^{\prime}$ with $\left\|p_{j}^{\prime}-p_{j}\right\|<\delta, j=1, \cdots, r$.

Finally, throughout this paper we will also let $S_{1}(H, k, N) \subset M \times H^{k-n-1}$ denote the set of $(k-n)$-tuples $\left(x, p_{n+2}, \cdots, p_{k}\right) \in M \times H^{k-n-1}$ so that

$$
\left(\xi(x), p_{n+2}, \cdots, p_{k}\right) \in S(H, k, N) \text {. }
$$

With $S_{1}(H, k, N)$ we associate the mapping $\Lambda_{1}: S_{1}(H, k, N) \rightarrow G(H, k, N)$ defined by

$$
\Lambda_{1}=\Lambda \circ\left(\xi \times\left(\mathrm{id}_{H}\right)^{k-n-1}\right) .
$$

Lemma 2.4. The maps $\Lambda_{1}: S_{1}(H, k, N) \rightarrow G(H, k, N)$ are smooth Fredbolm maps.

Proof. Clearly, letting $L_{0}=\Lambda_{1}\left(x, p_{n+2}, \cdots, p_{k}\right)-p$ where $p \in N$, or $p \epsilon$ $\Lambda_{1}\left(x, p_{n+2}, \cdots, p_{k}\right)$ if $N=\emptyset$, and letting $K, \Pi, \bar{\Pi}$ be defined just as before, we have, for $u \in T_{x}, u_{j} \in H, j=n+2, \cdots, k$,

$$
d \Lambda_{1}\left(u, u_{n+2}, \cdots, u_{k}\right)=\left(\bar{\Pi} d \xi(u), \bar{\Pi}\left(u_{n+2}\right), \ldots, \bar{\Pi}\left(u_{k}\right)\right) .
$$

Hence,

$$
\operatorname{ker} d \Lambda_{1}=\left(T_{x} \cap L_{0}\right) \times L_{0}^{k-n-1}, \quad \operatorname{im} d \Lambda_{1}=\left(\bar{\Pi} T_{x}\right) \times K^{k-n-1}
$$

so that

index $d \Lambda_{1}=\operatorname{dim} \operatorname{ker} d \Lambda_{1}-\operatorname{codimim} d \Lambda_{1}$

$$
=(k(k-n-1)+k-\epsilon)-(1-\epsilon)=k(k-n)-1 \text {. Q.E.D. }
$$

Corollary 2.5. If $L \in G(H, k, N)$ is transverse to $M$ at $x_{1}, \cdots, x_{s}$ then for given $\delta>0$ there exists a neighbourbood $V$ of $L$ so that, if $L^{\prime} \in V, L^{\prime}$ contains points $\xi\left(x_{1}^{\prime}\right), \ldots, \xi\left(x_{s}^{\prime}\right)$ with $x_{i}^{\prime} \in U_{\delta}\left(x_{i}\right), i=1, \ldots, s$, where $U_{\delta}\left(x_{i}\right)$ denotes the open geodesic $\delta$-ball about $x_{i}$.

Proof. For each $i=1, \cdots, s$ we have $L=\Lambda_{1}\left(x_{i}, q_{1}, \cdots, q_{n_{i}}\right)$ for some $q_{1}, \cdots, q_{n_{i}} \in H$, so that by (5) there is a neighbourhood $V_{i}$ of $L^{\prime}$ so that $L^{\prime} \in V_{i}$ $\Rightarrow L^{\prime}$ contains a point $\xi\left(x_{i}^{\prime}\right), x_{i}^{\prime} \in U_{\delta}\left(x_{i}\right)$. The corollary follows if we take $V^{i}=$ $\bigcap_{i=1}^{r} V_{i}$. Q.E.D.

Theorem 2.6. Let $N \in G(H, n), n<k$, be transverse to $M$. Then

$$
\{L \in G(H, k, N) \mid L \text { not transverse to } M\}
$$


is of the first category in $G(H, k, N)$.

Proof. Note that $L \in G(H, k, N)$ fails to be transverse to $M$ if and only if it is a singular value for $\Lambda_{1}: S_{1}(H, k, N) \rightarrow G(H, k, N)$. Since $\Lambda_{1}$ is a Fredholm map, this set of singular values is of the first category in $G(H, k, N)$ (see [8, Theorem 1.3]). Q.E.D.

In the case $N=p$, the condition that $N$ and $M$ be transverse is that $p \notin M$. We have the following stronger theorem for this case:

Theorem 2.7. For any $p \in H$, the set

$$
\{L \in G(H, k, p) \mid L \text { not transverse to } M\}
$$

is of the first category in $G(H, k, p)$.

Proof. As in the preceding theorem,

$$
X=\left\{L \in G(H, k, p) \mid L \text { not transverse to } M-\xi^{-1}(p)\right\}
$$

is of the first category. It remains to show that the set

$$
Y=\{L \in G(H, k, p) \mid L \text { not transverse to } M \text { at } p\}
$$

is of the first category. Since $M$ is immersed, the set $\xi^{-1}(p) \subset M$ is discrete; since $M$ is separable, $\xi^{-1}(p)$ must then be countable, say $\xi^{-1}(p)=\left\{x_{1}, x_{2}, \ldots\right\}$. Now $M$ and $L$ fail to be transverse at $p$ if and only if $L \subset T_{x_{j}}+p$ for some integer $j$. Let $Y_{j}$ be the set of $L \in G(H, k, p)$ such that $L \subset T_{x_{j}}+p$. This is clearly a nowhere dense set. Hence $Y=\bigcup_{j=1}^{\infty} Y_{j}$ is of the first category. Q.E.D.

3. The connected components of $\xi^{-1}(L)$. If $x \in M$, let $U_{\delta}(x)$ denote the geodesic $\delta$-ball about $x, U_{\delta}(x)=\{y \in M \mid d(y, x)<\delta\}$. The purpose of this section is to prove the following proposition:

Proposition 3.1. Let $x_{1}, \cdots, x_{r} \in M$. Then there is an integer $k$ and an $L \epsilon$ $G(H, k)$ so that $x_{1}, \cdots, x_{r}$ belong to the same connected component of $\xi^{-1}(L)$. Moreover, for each $\delta>0$ there is a neighbourbood $V$ of $L$ so that

$$
L^{\prime} \in V \Rightarrow \xi^{-1}\left(L^{\prime}\right) \cap U_{\delta}\left(x_{i}\right) \neq \varnothing \text { for } i=1, \ldots, r
$$

and so that, if $y_{i} \in \xi^{-1}\left(L^{\prime}\right) \cap U_{\delta}\left(x_{i}\right), i=1, \ldots, r$, then $y_{1}, \cdots, y_{r}$ belong to the same connected component of $\xi^{-1}\left(L^{\prime}\right)$.

To prove it we need a lemma. Let $\nu(x)$ denote a unit normal to $\xi(M)$ at $x \in M$.

Lemma 3.2. Each point $z_{0} \in M$ bas a neighbourbood $U \subset M$ together with a neighbourbood $B$ of $\nu\left(z_{0}\right)$ so that, for any $z_{1}, z_{2} \in U$ and $q \in \xi\left(z_{0}\right)+B, z_{1}$ and $z_{2}$ lie in the same connected component of $\xi^{-1}\left[\xi\left(z_{1}\right), \xi\left(z_{2}\right), q\right]$, and $\left[\xi\left(z_{1}\right), \xi\left(z_{2}\right)\right.$, $q]$ and $M$ are transverse at $z_{1}$ and $z_{2}$. 
Proof. Let $W$ be a neighbourhood of $\nu\left(z_{0}\right)$ in $S=\{\nu \in H \mid\|\nu\|=1\}$. For $\mu \in W$ let $\Pi_{\mu}: H \rightarrow T_{z_{0}}$ be the projection given by

$$
q=\Pi_{\mu}(q)+\lambda \mu, \quad \lambda \in \mathbf{R} .
$$

The lemma is proved if we can show that, for $W$ small enough and for $z_{1}, z_{2}$ belonging to a suitably small neighbourhood $U$ of $z_{0}$, the line segment joining $\Pi_{\mu}\left(\xi\left(z_{1}\right)\right)$ and $\Pi_{\mu}\left(\xi\left(z_{2}\right)\right)$ is the $\left(\Pi_{\mu} \circ \xi\right)$-image of a continuous curve in $M$.

Clearly $\Pi_{\mu}(q)$ depends differentiably on $(\mu, q) \in W \times H$. Define the differentiable map $\theta: W \times M \rightarrow W \times T_{z_{0}}$ by

$$
\theta(\mu, z)=\left(\mu, \Pi_{\mu}\left(\xi(z)-\xi\left(z_{0}\right)\right)\right)
$$

Since $d \theta$ is then given by

$$
d \theta=\mathrm{id}_{H} \times d \xi: H \times M_{z_{0}} \rightarrow H \times T_{z_{0}},
$$

there is an open geodesic $\epsilon$-ball $B_{\epsilon}^{\prime} \subset S$ with center $\nu\left(z_{0}\right)$ and a neighbourhood $U^{\prime}$ of $z_{0} \in M$ so that $\theta: B_{\epsilon}^{\prime} \times U^{\prime} \rightarrow B_{\epsilon}^{\prime} \times T_{z_{0}}$ is a diffeomorphism onto its image. In particular, for each $\mu \in B_{\epsilon}^{\prime}$,

$$
\Pi_{\mu} \circ\left(\xi-\xi\left(z_{0}\right)\right): U^{\prime} \rightarrow T_{z_{0}}
$$

is a diffeomorphism. Choose $U^{\prime}$ so that, for some $r>0$,

$$
\Pi_{\nu\left(z_{0}\right)}\left(\xi\left(U^{\prime}\right)-\xi\left(z_{0}\right)\right)=D(r),
$$

the open $r$-ball about $0 \in T_{z_{0}}$, with $r$ so small that, for $z \in U^{\prime}, \delta>0$,

$$
\left\|\Pi_{\nu\left(z_{0}\right)}\left(\xi(z)-\xi\left(z_{0}\right)\right)-\xi(z)+\xi\left(z_{0}\right)\right\|<\delta
$$

Now put

$$
U=\left(\mathrm{II}_{\nu\left(z_{0}\right)} \circ\left(\xi-\xi\left(z_{0}\right)\right) \mid U^{\prime}\right)^{-1}(D(r-2 \delta \tan \epsilon))
$$

and let $\mu \in B_{\epsilon}^{\prime}$. Let

$$
p_{t}=(1-t)\left(\xi\left(z_{1}\right)-\xi\left(z_{0}\right)\right)+t\left(\xi\left(z_{2}\right)-\xi\left(z_{0}\right)\right), \quad 0 \leq t \leq 1,
$$

and let $l_{t}, 0 \leq t \leq 1$, be the line $l_{t}(s)=s \mu+p_{t}, s \in \mathbf{R}$. It is enough to show that $l_{t}$ intersects $\xi\left(U^{\prime}\right)-\xi\left(z_{0}\right)$.

Let $\Pi_{0}$ be the function on $H$ given by

$$
p=\Pi_{\nu\left(z_{0}\right)}(p)+\Pi_{0}(p) \nu\left(z_{0}\right)
$$

Then

$$
\left\|\Pi_{\nu\left(z_{0}\right)}\left(l_{t}(s)\right)-\Pi_{\nu\left(z_{0}\right)}\left(p_{t}\right)\right\|<\tan \epsilon\left|\Pi_{0}\left(l_{t}(s)\right)-\Pi_{0}\left(p_{t}\right)\right|
$$


Let $f$ be the $C^{\infty}$ function defined on $\left(\Pi_{\nu\left(z_{0}\right)}\right)^{-1}(D(r))$ by

$$
f(p)=\Pi_{0}(p)-\Pi_{0}\left\{\left\{\xi \circ\left(\Pi_{\nu\left(z_{0}\right)} \circ\left(\xi-\xi\left(z_{0}\right)\right) \mid U^{\prime}\right)^{-1} \circ \Pi_{\nu\left(z_{0}\right)}\right\}(p)-\xi\left(z_{0}\right)\right\} .
$$

Restrict this function to $l_{t}$ to give $f \circ l_{t}: \mathbf{R} \rightarrow \mathbf{R}$. We claim that $f \circ l_{t}$ is defined for $|s| \leq 2 \delta / \cos \epsilon$; for then

$$
\left\|\Pi_{\nu\left(z_{0}\right)}\left(l_{t}(s)\right)-\Pi_{\nu\left(z_{0}\right)}\left(p_{t}\right)\right\| \leq 2 \delta \tan \epsilon
$$

which combines with $\Pi_{\nu\left(z_{0}\right)}\left(p_{t}\right) \in D(r-2 \delta \tan \epsilon)$ to give $\Pi_{\nu\left(z_{0}\right)}\left(l_{t}(s)\right) \in D(r)$. But

$$
\begin{gathered}
\left|\Pi_{0}\left(p_{t}\right)\right|<\delta, \\
\left|\Pi_{0}\left(l_{t}( \pm 2 \delta / \cos \epsilon)\right)-\Pi_{0}\left(p_{t}\right)\right|>2 \delta, \\
\left|\Pi_{0}\left\{\xi \circ\left[\Pi_{\nu\left(z_{0}\right)} \circ\left(\xi-\xi\left(z_{0}\right)\right) \mid U^{\prime}\right]^{-1} \circ \Pi_{\nu\left(z_{0}\right)}\right\}\left(l_{t}(2 \delta / \cos \epsilon)\right)-\xi\left(z_{0}\right)\right|<\delta
\end{gathered}
$$

together yield

$$
f \circ l_{t}(2 \delta / \cos \epsilon)>0, \quad f \circ l_{t}(-2 \delta / \cos \epsilon)<0 .
$$

By the intermediate value theorem this shows that $l_{t}$ intersects $\xi\left(U^{\prime}\right)-\xi\left(z_{0}\right)$. Q.E.D.

Proof of the proposition. By joining the points $x_{1}, \ldots, x_{r}$, with a curve in $M$ and covering the curve by finitely many neighbourhoods of the kind discussed in Lemma 3.2, we can arrange to have the following situation: $\left\{x_{1}, \ldots, x_{r}\right\}$ is included in a finite set $\left\{z_{1}, \cdots, z_{s}\right\} \subset M$ such that, for each $i=1, \cdots, s-1, z_{i}$ and $z_{i+1}$, together with a point $q_{i} \in H$ and neighbourhoods $U_{i} \subset M$ containing $z_{i}$ and $z_{i+1}$ and $B_{i} \subset H$ containing $q_{i}$, satisfy the conclusion of Lemma 3.2. That is, if

$$
L_{i}=\left[\xi\left(z_{i}^{\prime}\right), \xi\left(z_{i+1}^{\prime}\right), q_{i}^{\prime}\right], \quad z_{i}^{\prime}, z_{i+1}^{\prime} \in U_{i}, q_{i}^{\prime} \in B_{i},
$$

then $z_{i}^{\prime}$ and $z_{i+1}^{\prime}$ lie in the same connected component of $\xi^{-1}\left(L_{i}^{\prime}\right)$, and $L_{i}^{\prime}$ and $M$ are transverse at $z_{i}^{\prime}$ and $z_{i+1}^{\prime}$. Clearly, if we put $L_{i}=\left[\xi\left(z_{i}\right), \xi\left(z_{i+1}\right), q_{i}\right], i=$ $1, \cdots, s-1$, and $L=\left[L_{1}, \cdots, L_{s-1}\right]$, then $x_{1}, \cdots, x_{r}$ lie in the same connected component of $\xi^{-1}(L)$. By Corollary 2.5, $L$ has a neighbourhood $V$ so that $L^{\prime} \in V$ implies that

$$
L^{\prime} \cap \xi\left(U_{i} \cap U_{i-1} \cap U_{\delta}\left(z_{i}\right)\right) \neq \emptyset
$$

Take

$$
z_{i}^{\prime} \in U_{i} \cap U_{i-1} \cap U_{\delta}\left(z_{i}\right) \cap \xi^{-1}\left(L^{\prime}\right), \quad i=1, \ldots, s .
$$

Then $z_{1}^{1}, \ldots, z_{s}^{\prime}$ are all in the same connected component of $\xi^{-1}\left(L^{\prime}\right)$. Among these points $z_{1}^{\prime}, \ldots, z_{s}^{\prime}$ we can find the points $y_{1}, \ldots, y_{r}$ to satisfy the conclusion of the proposition. Q.E.D. 
4. The curvature of a transverse linear section. In this section we first show that, if $L$ is transverse to $M, \xi^{-1}(L)$ is a submanifold of $M$ (Proposition 4.1). We then show that the sign of a sectional curvature of $\xi^{-1}(L)$ is the same as the sign of the corresponding sectional curvature of $M$ (Proposition 4.2). We complete the section by showing that in some cases the sectional curvatures of $\xi^{-1}(L)$ depend smoothly on $L$ (Lemma 4.3).

Proposition 4.1. Let $L \in G(H, k)$ be transverse to $M$. Then $\xi^{-1}(L)$ is an (embedded) $C^{\infty}$ submanifold of $M$.

Proof. It suffices to find Hilbert spaces $E_{1}, E_{2}, E=E_{1} \oplus E_{2}$, and open sets $U_{1} \subset E_{1}, U_{2} \subset E_{2}$ and a chart $\Psi: E=E_{1} \oplus E_{2} \supset U_{1} \times U_{2} \rightarrow M$ centered at $x \in M$ so that

$$
\Psi\left(U_{1} \times 0\right)=\Psi\left(U_{1} \times U_{2}\right) \cap \xi^{-1}(L) .
$$

Let $(E \supset U, \phi)$ be any chart for $M$ centered at $x$. Let $K$ be the orthogonal complement of $L$ and note that

$$
L+d(\xi \phi)_{0}(E)=L+T_{x}=H
$$

since $M$ and $L$ are transverse. Hence, if $\bar{\Pi}: H \rightarrow K$ is the orthogonal projection, $\bar{\Pi} \circ d(\xi \phi)_{0}: E \rightarrow K$ is onto. Let $N \subset E$ be the kernel of $\bar{\Pi} \circ d(\xi \phi)_{0}$ and $P$ its orthogonal complement. Then the partial derivative with respect to $P$ at $0 \in E$ is given by

$$
d_{2}(\bar{\Pi} \xi \phi)_{0}=\bar{\Pi} \circ\left(d(\xi \phi)_{0} \mid P\right): P \rightarrow K .
$$

By the inverse function theorem (see [4]) there exist closed subspaces $E_{1}$ and $E_{2}$ of $E=E_{1} \oplus E_{2}$ with open sets $U_{1} \subset E_{1}, U_{2} \subset E_{2}$ containing the origin and a diffeomorphism $b$ of $U_{1} \times U_{2}$ onto a nieghbourhood of 0 contained in $U$ so that $\bar{\Pi} \circ \xi \circ \phi \circ b: U_{1} \times U_{2} \rightrightarrows K$ is a projection onto $U_{2}$. Put $\Psi=\phi \circ b$. Then for $\left(x_{1}, x_{2}\right) \in U_{1} \times U_{2}$ we have

That is,

$$
\xi \circ \Psi\left(x_{1}, x_{2}\right) \in L \Leftrightarrow \bar{\Pi} \xi \phi b\left(x_{1}, x_{2}\right)=0 \Leftrightarrow x_{2}=0 .
$$

$$
\Psi\left(U_{1} \times 0\right)=\Psi\left(U_{1} \times U_{2}\right) \cap \xi^{-1}(L) \text { Q.E.D. }
$$

Proposition 4.2. Let $L \in G(H, k)$ be transverse to $M$. For a plane section $\sigma$ tangent to $\xi^{-1}(L) \subset M$ let $\bar{K}_{\sigma}$ and $K_{\sigma}$ denote the sectional curvatures of $\xi^{-1}(L)$ at $\sigma$ and $M$ at $\sigma$ respectively. Then eitber $\bar{K}_{\sigma}$ and $K_{\sigma}$ are both zero, or else they are both nonzero and have the same sign.

Proof. Let $R$ and $\bar{R}$ be the curvature tensors on $M$ and $\xi^{-1}(L)$ respectively, and let $\nabla$ and $\bar{\nabla}$ be the connections on $H$ and $L$, and let $\nu$ and $\bar{\nu}$ be (local) unit normal fields to $\xi(M)$ in $H$ and to $\xi\left(\xi^{-1}(L)\right)$ in $L$. For $u, v \in\left(\xi^{-1}(L)\right)_{x} C$ $M_{x}$ we have the following form of Gauss's Theorema Egregium: 


$$
\begin{aligned}
& \langle R(u, v) v, u\rangle=\left\langle\nabla_{u} u, \nu\right\rangle\left\langle\nabla_{v} v, \nu\right\rangle-\left\langle\nabla_{u} v, \nu\right\rangle^{2}, \\
& \langle\bar{R}(u, v) v, u\rangle=\left\langle\bar{\nabla}_{u} u, \bar{\nu}\right\rangle\left\langle\bar{\nabla}_{v} v, \bar{\nu}\right\rangle-\left\langle\bar{\nabla}_{u} v, \bar{\nu}\right\rangle^{2} .
\end{aligned}
$$

Let $x \in \xi^{-1}(L)$ and let $\sigma \subset\left(\xi^{-1}(L)\right)_{x}$ be a plane section. We may choose $u$ and $v$ to be vector fields tangent to $M$ in an $M$-neighbourhood $U$ of $x$ so that $\sigma$ is generated by $u(x)$ and $v(x)$ and so that $u$ and $v$ are tangent to $\xi^{-1}(L)$ at all points of $\xi^{-1}(L) \cap U$, and so that $[u, v]=0$ on $\xi^{-1}(L) \cap U$. If $K$ is the orthogonal complement of $L$ we have $\nu=\nu_{1}+\nu_{2}, \nu_{1} \in L, \nu_{2} \in K$, and because $T_{x}$ and $L$ are transverse, $\left\|\nu_{1}\right\| \neq 0$. Then $\bar{\nu}=\nu_{1} /\left\|\nu_{1}\right\|$, so that, by (7),

$$
\begin{aligned}
& \left(1 /\left\|\nu_{1}\right\|\right)^{2}\left\{\left\langle\bar{\nabla}_{u} u, \nu_{1}\right\rangle\left\langle\bar{\nabla}_{v} v, \nu_{1}\right\rangle-\left\langle\bar{\nabla}_{u} \nu, \nu_{1}\right\rangle^{2}\right\} \\
& \quad=\left(1 /\left\|\nu_{1}\right\|\right)^{2}\left\{\left\langle\nabla_{u} u, \nu\right\rangle\left\langle\nabla_{\nu} v, \nu\right\rangle-\left\langle\nabla_{u} v, \nu\right\rangle^{2}\right\}=\left(1 /\left\|\nu_{1}\right\|\right)^{2}\langle R(u, v) v, u\rangle,
\end{aligned}
$$

since by the choice of $u$ and $v, \nabla_{u} u, \nabla_{v} v$ and $\nabla_{u} v$ are automatically tangent to L. Q.E.D.

Remark. The fact that $L$ is finite dimensional is not used in the proofs of Propositions 4.1 or 4.2 . Both results are true if $L$ is any closed linear submanifold of $H$, transverse to $M$.

Lemma 4.3. Suppose that $\left\{\xi(x), p_{1}, \cdots, p_{k}\right\}$ is in general position and that $L=\left[\xi(x), p_{1}, \cdots, p_{k}\right]$ is transverse to $M$ at $x \in \xi^{-1}(L)$ and that at least one sectional curvature at $x$ in $\xi^{-1}(L)$ is positive. Then for some $\delta>0$ this remains true at $y$ for $\left[\xi(y), q_{1}, \cdots, q_{k}\right]$ whenever $y \in U_{\delta}(x)$ and $q_{j} \in B_{\delta}\left(p_{j}\right), j=1, \cdots, k$.

Proof. If $\delta$ is small enough, $\left\{\xi(y), q_{1}, \cdots, q_{k}\right\}$ is automatically again in general position. Then

$$
\left\{\Lambda_{1}\left(y, q_{1}, \cdots, q_{k}\right)-\xi(y)\right\} \cap T_{y}
$$

is the orthogonal complement in $\Lambda_{1}\left(y, q_{1}, \cdots, q_{k}\right)-\xi(y)$ of the projection of the unit normal $\nu(y)$ on $\Lambda_{1}\left(y, q_{1}, \cdots, q_{k}\right)-\xi(y)$. That projection is given by

$$
\bar{\nu}\left(y, q_{1}, \cdots, q_{k}\right)=\sum_{j=1}^{k}\left\langle\nu(y), q_{j}-\xi(y)\right\rangle g^{j k}\left(q_{k}-\xi(y)\right),
$$

where $\left(\left(g^{j k}\right)\right)=\left(\left(g_{j k}\right)\right)^{-1}$, and $g_{j k}=\left\langle q_{j}-\xi(y), q_{k}-\xi(y)\right\rangle$. This shows that $\bar{\nu}\left(y, q_{1}, \cdots, q_{k}\right)$ varies smoothly with $\left(y, q_{1}, \cdots, q_{k}\right)$. In particular, if $\delta$ is small enough,

$$
\left\|\bar{\nu}\left(y, q_{1}, \cdots, q_{k}\right)\right\| \neq \varnothing,
$$

so that $\Lambda_{1}\left(y, q_{1}, \cdots, q_{k}\right)$ and $M$ are transverse at $y$. It also follows that $\mu=$ $\bar{\nu} /\|\bar{\nu}\|$ is smooth in its arguments. But a set of generators for $\left\{\Lambda_{1}\left(y, q_{1}, \cdots, q_{k}\right)-\right.$ $\xi(y)\} \cap T_{y}$ is then given by

$$
w_{j}\left(y, q_{1}, \cdots, q_{k}\right)=q_{j}-\xi(y)-\left\langle q_{j}-\xi(y), \mu\right\rangle \mu, \quad j=1, \cdots, k,
$$

which also vary smoothly. Hence, so do the sectional curvatures 


$$
K(u, v)=\langle\bar{R}(u, v) v, u\rangle /\left\{\|u\|^{2}\|v\|^{2}-\langle u, v\rangle^{2}\right\}
$$

when, for $a^{i}, b^{j} \in \mathbf{R}$,

$$
\begin{aligned}
& u=u\left(y, q_{1}, \cdots, q_{k}\right)=\sum_{i=1}^{k} a^{i} w_{i}\left(y, q_{1}, \cdots, q_{k}\right), \\
& v=v\left(y, q_{1}, \cdots, q_{k}\right)=\sum_{j=1}^{k} b^{j} w_{j}\left(y, q_{1}, \cdots, q_{k}\right) .
\end{aligned}
$$

Hence, if $a^{i}$ and $b^{j}$ are chosen in such a way that

then also

$$
K\left(u\left(x, p_{1}, \cdots, p_{k}\right), v\left(x, p_{1}, \cdots, p_{k}\right)\right)>0,
$$

$$
K\left(u\left(y, q_{1}, \cdots, q_{k}\right), v\left(y, q_{1}, \cdots, q_{k}\right)\right)>0,
$$

if, for a small enough $\delta>0$,

$$
y \in U_{\delta}(x), \quad q_{j} \in B_{\delta}\left(p_{j}\right), \quad j=1, \cdots, k . \quad \text { Q.E.D. }
$$

5. Hypersurfaces with positive sectional curvatures. In this section we prove the following generalization of the theorem of Sacksteder $[7$, Theorem. $(*)]$ which we shall refer to as $(S)$ for convenience.

Theorem 5.1. Let $M$ be a complete Riemannian Hilbert manifold with nonnegative sectional curvatures and with at least one point at which at least one sectional curvature is strictly positive. Let $\xi: M \rightarrow H$ be an isometric immersion of $M$ as a bypersurface of a Hilbert space $H$. Then

(i) $\xi$ is an embedding.

(ii) $\xi(M) \subset H$ is the boundary of a convex body in $H$; in particular, $\xi$ embeds $M$ topologically as a closed subset of $H$.

(iii) $M$ is bomeomorpbic to $H \times S^{n}$ for some integer $n \geq 0$.

Proof. We shall let $x_{0}$ and $\sigma \subset M_{x_{0}}$ be a point and a plane section at $x_{0}$ at which the sectional curvature is strictly positive.

(a) Suppose first that $\xi$ is not one-to-one. Say $\xi\left(y_{1}\right)=\xi\left(y_{2}\right)=q \in H$. By Proposition 3.1 there is an $L \in G(H, l, q)$ transverse to $M$ at $x_{0}$ together with a neighbourhood $V$ of $L$ in $G(H, l, q)$ so that, if $L^{\prime} \in V, L^{\prime}$ contains $\xi(x)$ for some $x \in U_{\epsilon}\left(x_{0}\right)$, and $y_{1}, y_{2}, x$ lie in the same connected component of $\xi^{-1}\left(L^{\prime}\right)$. We may assume that $d \xi(\sigma)+\xi\left(x_{0}\right) \subset L$, and then by Lemma 4.3 we may assume that at $x \in \xi^{-1}\left(L^{\prime}\right)$ there is a plane section of strictly positive curvature. By Theorem 2.7 we may assume $L^{\prime}$ and $M$ to be transverse. But then we can apply (S) to the connected component of $\xi^{-1}\left(L^{\prime}\right)$ containing $x$, to get a contradiction to $\xi\left(y_{1}\right)=$ $\xi\left(y_{2}\right)$.

(b) We now show that no line intersecting $\xi(M)$ transversally twice intersects it again. For suppose there is a line that intersects $M$ transversally at $\xi\left(x_{1}\right)$ and $\xi\left(x_{2}\right)$ and intersects $M$ again at $\xi\left(x_{3}\right)$. Then for $y_{1}$ sufficiently near $x_{1}$ on $M$, 
the line $\left[\xi\left(y_{1}\right), \xi\left(x_{3}\right)\right]$ will also be transverse to $M$ at $y_{1}$ and at a point $y_{2}$ near $x_{2}$. By applying Proposition 3.1, Corollary 2.5, Theorem 2.7, and Lemma 4.3 in that order, we may suppose that $\left[\xi\left(y_{1}\right), \xi\left(x_{3}\right)\right]$ is contained in a linear submanifold $L \in G\left(H, l, \xi\left(x_{3}\right)\right)$ transverse to $M$, so that, for some $x$ near $x_{0}, y_{1}, y_{2}, y_{3}, x$ are contained in the same connected component $X$ of $\xi^{-1}(L)$, and so that at least one sectional curvature of $\xi^{-1}(L)$ at $x$ is strictly positive. But then $\xi: X \rightarrow L$ satisfies (S), contradicting the assumption that $\left[\xi\left(y_{1}\right), \xi\left(y_{2}\right)\right]$ meets $\xi\left(\xi^{-1}(L)\right)$ a third time.

(c) To show now that $\xi$ is an embedding it is enough to show that each $y_{0} \epsilon$ $M$ has an arbitrarily small neighbourhood $U$ so that, for some neighbourhood $V C$ $H$ of $\xi\left(y_{0}\right), \xi^{-1}(V)=U$.

If $\xi(M)$ is contained in $T_{y_{0}}+\xi\left(y_{0}\right)$, this is trivial. Otherwise, let $f$ be defined on $M-y_{0}$ by

$$
f(y)=\left\|\xi(y)-\xi\left(y_{0}\right)\right\| \text {. }
$$

Let $y \in M$ be such that $\xi(y) \notin T_{y_{0}}+\xi\left(y_{0}\right)$. By following the integral trajectory $a$ of - $\operatorname{grad} /$ and using (a) and the completeness of $M$ we see that $\alpha$ must contain a point $y_{1}$ such that $\xi\left(y_{1}\right) \notin T_{y_{0}}+\xi\left(y_{0}\right)$ and such that $M$ and $\left[\xi\left(y_{0}\right), \xi\left(y_{1}\right)\right]$ are transverse at $y_{1}$. Choose neighbourhoods $U$ of $y_{0}$ and $W$ of $y_{1}$ such that whenever $y_{0}^{\prime} \in U, y_{1}^{\prime} \in W$; then $\left[\xi\left(y_{0}^{\prime}\right), \xi\left(y_{1}^{\prime}\right)\right]$ and $M$ are transverse at $y_{1}^{\prime}$ and $y_{0}^{\prime}$. Put

$$
v=\left\{t \xi\left(y_{1}^{\prime}\right)+(1-t) \xi\left(y_{0}^{\prime}\right) \mid t \in(-1,1), y_{0}^{\prime} \in U, y_{1}^{\prime} \in W\right\} \text {. }
$$

If $V$ contained points $\xi(y), y \notin U$, (b) would be contradicted. This completes the proof of (i).

(d) We will now show that the normal bundle over $M$ is trivial. If this is not the case, there is a curve $\gamma(t)$ in $M$ so that $\gamma(0)=\gamma(1)=y_{0}$ and such that, if $\nu(\gamma(t))$ is a continuous lift of $\gamma$ to the unit normal bundle, $\nu(\gamma(0))=-\nu(\gamma(1))$. Use (c) to cover $M$ by an open covering $\left\{U_{\alpha}\right\}$ so that $U_{\alpha}=\xi^{-1}\left(V_{\alpha}\right)$ for a connected open set $V_{\alpha}$ $C H$. Let $V=U_{a} V_{a}$. Since $\gamma([0,1])$ is compact there is an $\epsilon>0$ so that $\beta(t)=$ $\xi(\gamma(t))+\epsilon \nu(\gamma(t)) \epsilon V-\xi(M), 0 \leq t \leq 1$. Approximate $\beta$ by a piecewise linear curve in $V-\xi(M)$ with the same end points, and combine it with the segment $\{\xi(\gamma(0))+$ $\epsilon t \nu(\gamma(0)) \mid-1 \leq t \leq 1\}$. This gives a closed piecewise linear curve in $V$ that intersects $\xi(M)$ transversely precisely once at $\xi\left(y_{0}\right)$. But then by Proposition 3.1, Theorem 2.7, and Lemma 4.3 there is an $L \in G\left(H, l, \xi\left(x_{0}\right)\right)$ transverse to $M$ with the following properties: (1) for some $y$ near $y_{0}, y$ and $x_{0}$ belong to the same connected component of $\xi^{-1}(L)$; (2) at least one sectional curvature of $\xi^{-1}(L)$ at $x_{0}$ is strictly positive; and (3) $V \cap L$ contains a piecewise linear curve that crosses $\xi\left(\xi^{-1}(L)\right)$ precisely once at $\xi(y)$. But this contradicts (S).

(e) Let $C$ be the convex hull of $\xi(M)$. We want to show that $\xi(M) \subset \partial C$. Suppose to the contrary that $\xi\left(y_{0}\right) \in$ int $C$. Then $C$, and therefore also $M$, lies on both sides of $T_{y_{0}}+\xi\left(y_{0}\right)$. Using precisely the same argument as that given in (c) 
there must be open sets $W_{1}, W_{2} \subset M$ and a neighbourhood $U$ of $y_{0}$ with the following property: If $y_{1}^{\prime} \in W_{1}$ and $y_{2}^{\prime} \in W_{2}$, and $y_{0}^{\prime} \in U$, then $\xi\left(y_{1}^{\prime}\right)$ and $\xi\left(y_{2}^{\prime}\right)$ lie on opposite sides of $T_{y_{0}^{\prime}}+\xi\left(y_{0}^{\prime}\right)$, and $\left[\xi\left(y_{0}^{\prime}\right), \xi\left(y_{1}^{\prime}\right)\right]$ and $\left[\xi\left(y_{0}^{\prime}\right), \xi\left(y_{2}^{\prime}\right)\right]$ are transverse to $M$ at $y_{0}^{\prime}$ and $y_{1}^{\prime}$ and at $y_{0}^{\prime}$ and $y_{2}^{\prime}$ respectively. By Proposition 3.1, Theorem 2.7 and Lemma 4.3 we may assume that $\left[\xi\left(y_{0}^{\prime}\right), \xi\left(y_{1}^{\prime}\right)\right]$ and $\left[\xi\left(y_{0}^{\prime}\right), \xi\left(y_{2}^{\prime}\right)\right]$ are included in an $L \in G\left(H, l, \xi\left(x_{0}\right)\right)$ which is transverse to $M$ and such that $x_{0}$, $y_{0}^{\prime}, y_{1}^{\prime}, y_{2}^{\prime}$ are contained in the same connected component of $\xi^{-1}(L)$ and so that $\xi^{-1}(L)$ has at least one section of strictly positive curvature at $x_{0}$. We get a contradiction now if we apply (S) to this connected component.

(f) $\xi(M)$ is an open subset of $\partial C$. It is clear that, since one sectional curvature is strictly positive at $x_{0}, C$ has a nonempty interior. Let $p$ be any interior point. Join $p$ to a point $\xi\left(y_{0}\right) \in \xi(M)$. The line $\left[p, \xi\left(y_{0}\right)\right]$ must be transverse to $M$ at $y_{0}$, for otherwise $\xi\left(y_{0}\right)$ could not be a boundary point of $C$. Hence for some neighbourhood $U \subset M$ of $y_{0}$ this remains true for $[p, \xi(y)], y \in U$. Thus the joins of $p$ to the points $\xi(y), y \in U$, form an open pencil of rays at $p$ which must intersect $\partial C$ in an open set, which clearly can be none other than $\xi(U)$.

(g) To prove that $\xi(M)=\partial C$ we borrow the following argument from [1]: Suppose $p \in \partial C-\xi(M)$. By [3, p. 31], $\partial C$ is connected, and thus there exists a rectifiable curve $\theta(t)$ in $\partial C, t \in[0,1]$ with $\theta(0)=\xi\left(y_{0}\right)$ and $\theta(1)=p$. Since $\xi(M)$ is open in $\partial C$ there must be some $t_{0} \in(0,1]$ so that $\theta(t) \in \xi(M)$ if $t<t_{0}$, while $\theta\left(t_{0}\right) \notin \xi(M)$. Let $\left\{t_{i}\right\}$ be a sequence approaching $t_{0}$ from below. Let $a_{i}$ be the arclength of $\theta$ between $\theta(0)$ and $\theta\left(t_{i}\right)$. Then $\left\{a_{i}\right\}$ converges and

$$
d\left(\theta\left(t_{i}\right), \theta\left(t_{j}\right)\right) \leq\left|a_{i}-a_{j}\right|
$$

shows that $\left\{\xi^{-1} \theta\left(t_{i}\right)\right\}$ is a Cauchy sequence in $M$ that does not converge, contradicting the completeness of $M$. The proof of (ii) is now also complete.

(h) Part (iii) is now a direct consequence of [3, p. 31, Proposition 1.7]. Q.E.D.

6. Hypersurfaces with zero sectional curvatures. In this section we prove the following generalization of the theorem of Hartman and Nirenberg [2, Theorem III] which we shall refer to as $(\mathrm{H})$ throughout this section.

Theorem 6.1. Let $M$ be a complete Riemannian Hilbert manifold with zero sectional curvatures. Let $\xi: M \rightarrow H$ be an isometric immersion of $M$ as a bypersurface of a Hilbert space $H$. Then $\xi(M)$ is a cylinder of the form $\beta \times A$ where $\beta$ is a plane curve and $A$ is the closed subspace of $H$ of codimension two orthogonal to the plane containing $\beta$.

Proof. Choose $x_{0} \in M$ and let $L$ be a plane through $\xi\left(x_{0}\right)$ transverse to $M$. If for each such $L$ the component of $\xi^{-1}(L)$ containing $x_{0}$ maps into a line, the theorem is finished. For then $\xi^{-1}(L)$ must be a geodesic, and thus for a dense subset of the unit tangent space $M_{x_{0}}(1)$, the corresponding geodesics would be 
mapped into lines by $\xi$. By the continuity of $\xi$ this would then be true for all $v \epsilon$ $M_{x_{0}}(1)$, making $\xi(M)$ a hyperplane in $H$.

Thus, we assume for the rest of the proof that we have a plane $L_{0}$ through $x_{0}$ transverse to $M$ so that the component of $\xi^{-1}\left(L_{0}\right)$ containing $x_{0}$ maps into a unit speed curve $a$ which is not a line.

Let $d \xi\left(v_{0}\right)$ be the velocity of $\alpha$ at $x_{0}, v_{0} \in M_{x_{0}}(1)$. If $L \in G\left(H, 3, L_{0}\right)$ is transverse to $M$, (H) tells us that $\xi\left(\xi^{-1}(L)\right)$ is a two dimensional cylinder generated by $\alpha$ and $d \xi(v)$ for some $v \in M_{x_{0}}(1)$. Let $K_{x_{0}} \subset M_{x_{0}}$ be the linear span of all vectors $v$ obtained this way. Clearly, $d \xi\left(K_{x_{0}}\right)$ is independent of the position of $x_{0}$ on $\alpha$. We want to show that if $v \in K_{x_{0}}$, then

$$
\xi \gamma_{\nu}(t)=\xi\left(x_{0}\right)+t d \xi(\nu),
$$

where $\gamma_{v}(t)$ denotes the geodesic in $M$ with $\gamma_{v}^{\prime}(0)=v$. Note that

$$
v=\sum_{i=1}^{n} a^{i} v_{i}, \quad v_{i} \in M_{x_{0}}(1),
$$

where $v_{i}$ is obtained as above as the generator of a cylinder $\xi\left(\xi^{-1}\left(L_{i}\right)\right), L_{i} \epsilon$ $G\left(H, 3, L_{0}\right)$ transverse to $M$. We may assume $v_{1}, \cdots, v_{n}$ linearly independent, and let $L^{\prime}=\left[L_{1}, \cdots, L_{n}\right]$. Let $\left\{L_{j}^{\prime}\right\}_{j=1}^{\infty}$ be an approximating sequence for $L^{\prime}$, $L_{j}^{\prime} \in G\left(H, n+2, L_{0}\right)$ transverse to $M$. Such a sequence exists by Theorem 2.6. For each $j$, the connected component of $\xi^{-1}\left(L_{j}^{\prime}\right)$ containing $x_{0}$ maps into an $(n+1)$-dimensional cylinder generated by $d \xi\left(v_{0}\right)$ and an $n$-space $d \xi\left(K_{j}\right), K_{j} \subset$ $M_{x_{0}}$. Select an orthonormal basis for $K_{j}$ :

$$
v_{j 1}, \cdots, v_{j n} \in K_{j} \cap M_{x_{0}}(1) .
$$

Let $w_{j 1}, \cdots, w_{j n}$ be the orthogonal projections of the vectors $d \xi\left(v_{j 1}\right), \ldots, d \xi\left(v_{j n}\right)$ on $L^{\prime}-\xi\left(x_{0}\right)$. For large $j$ these vectors will be linearly independent and will be close to unit lenght. Hence, by choosing a subsequence if necessary, we may arrange that, as $j \rightarrow \infty$,

$$
d \xi\left(v_{j i}\right) \rightarrow w_{i} \in L^{\prime}-\xi\left(x_{0}\right) .
$$

But then $\left\{v_{j i}\right\}_{j=1}^{\infty}$ is a Cauchy sequence in $M_{x_{0}}(1)$, whence there are vectors $u_{i} \epsilon$ $M_{x_{0}}(1), i=1, \cdots, n$, so that $v_{j i} \rightarrow u_{i}$ as $j \rightarrow \infty$. Clearly then $d \xi\left(u_{i}\right)=w_{i}$. But then

$$
\begin{aligned}
\xi \circ \gamma_{\Sigma a^{i} u_{i}}(t) & =\lim _{j \rightarrow \infty} \xi \circ \gamma_{\Sigma a^{i} v_{j i}}(t) \\
& =\lim _{j \rightarrow \infty}\left\{\xi\left(x_{0}\right)+t a^{i} d \xi\left(v_{j i}\right)\right\}=\xi\left(x_{0}\right)+t a^{i} w_{i}
\end{aligned}
$$

Thus the vectors $w_{1}, \cdots, w_{n}$ generate an $n$-dimensional linear subspace $J$ of $L^{\prime}$ $-\xi\left(x_{0}\right)$ with the property $J+\xi\left(x_{0}\right) \subset \xi(M)$. For any value of $i=1, \ldots, n$,

$$
\operatorname{dim}\left(L_{i}-\xi\left(x_{0}\right)\right)+\operatorname{dim} J=n+3=\operatorname{dim}\left(L^{\prime}-\xi\left(x_{0}\right)\right)+1,
$$


whence $J \cap\left\{L_{i}-\xi\left(x_{0}\right)\right\}$ must be at least one dimensional. In fact, since $J \subset \xi(M)$ $-\xi\left(x_{0}\right)$ and since the connected component of $\xi^{-1}\left(L_{i}\right)$ containing $x_{0}$ satisfies $(\mathrm{H})$, it follows that $J \cap\left\{L_{i}-\xi\left(x_{0}\right)\right\}$ is precisely the line generated by $d \xi\left(v_{i}\right)$. Thus $d \xi\left(v_{1}\right), \ldots, d \xi\left(v_{n}\right)$ will also generate $J$, whence, for $v \in K_{x_{0}}, \xi \circ \gamma_{v}$ is a straight line.

If $v \in \bar{K}_{x_{0}}, \xi \circ \gamma_{v}$ is a straight line. For let $v_{i} \in K_{x_{0}}$ be such that $v_{i} \rightarrow v$ as $i \rightarrow \infty$. Then

$$
\begin{aligned}
\xi \circ \gamma_{v}(t) & =\lim _{i \rightarrow \infty} \xi \circ \gamma_{v_{i}}(t) \\
& =\lim _{i \rightarrow \infty}\left\{\xi\left(x_{0}\right)+t d \xi\left(v_{i}\right)\right\}=\xi\left(x_{0}\right)+t d \xi(v) .
\end{aligned}
$$

It also follows that $v_{0} \notin \bar{K}_{x_{0}}$, for otherwise $\xi \circ \gamma_{v_{0}}$ would be a straight line, which would mean $\xi \circ \gamma_{v_{0}} \subset L_{0}$. But then $\xi \circ \gamma_{\nu_{0}}(t)=\alpha(t)$ which contradicts our assumption that $\alpha$ is not a straight line.

We want to prove now that $v_{0}$ and $\bar{K}_{x_{0}}$ together span $M_{x_{0}}$. Suppose there is a vector $v \in M_{x_{0}}(1)$ orthogonal to both $v_{0}$ and $\bar{K}_{x_{0}}$. Let $\epsilon>0$. By Theorem 2.6 there is a linear manifold $L \in G\left(H, 3, L_{0}\right)$ transverse to $M$ and so that there is a vector

By (H), $w$ may be expressed as

$$
w \in(d \xi)^{-1}\left(L-\xi\left(x_{0}\right)\right) \cap M_{x_{0}}(1), \quad\langle w, v\rangle^{2}>1-\epsilon^{2}>0
$$

$$
w=a v_{0}+b w^{\prime}, \quad w^{\prime} \in \bar{K}_{x_{0}} \cap M_{x_{0}}(1), \quad a, b \in \mathbf{R} .
$$

But then $\left\langle v, v_{0}\right\rangle=0=\left\langle v, w^{\prime}\right\rangle$ implies also $\langle v, w\rangle=0$ which contradicts $|\langle v, w\rangle|$ $>0$.

Theorem 6.1 now follows if we put $A=d \xi\left(\bar{K}_{x_{0}}\right), B=$ the orthogonal complement of $A$, and $\beta$ the orthogonal projection of $\alpha$ on $B$. Q.E.D.

\section{BIBLIOGRAPHY}

1. M. P. doCarmo, Positively-curved hypersurfaces of a Hilbert space, J. Differential Geometry 2 (1968), 355-362. MR 40 \#8081.

2. P. Hartman and L. Nirenberg. On spherical image maps whose Jacobians do not change sign, Amer. J. Math. 81 (1959), 901-920. MR 23 \#A4106.

3. V. Klee, Convex bodies and periodic homeomorphisms in Hilbert space, Trans. Amer. Math. Soc. 74 (1953), 10-43. MR 14,989.

4. S. Lang, Introduction to differentiable manifolds, Interscience, New York, 1962. MR 27 \#5192.

5. J. McAlpin, Infinite dimensional manifolds and Morse theory, Thesis, Columbia University, New York, 1965.

6. A. V. Pogorelov, Continuous maps of bounded variation, Dokl. Akad. Nauk SSSR 111 (1956), 757-759. (Russian) MR 19, 309; Extensions of the theorem of Gauss on spher$i c a l$ representation to the case of surfaces of bounded extrinsic curvature, Dokl. Akad. Nauk SSSR 111 (1956), 945-947. (Russian) MR 19, 309.

7. R. Sacksteder. On hypersurfaces with no negative sectional curvatures, Amer. J. Math. 82 (1960), 609-630. MR 22 \#7087.

8. S. Smale. An infinite dimensional version of Sard's theorem, Amer. J. Math. 87 (1965), 861-866. MR $32 \# 3067$. 